\title{
Evolution of a Cylindrical and a Spherical Vortex Sheet
}

\author{
Monika Nitsche \\ Institute for Mathematics and its Applications \\ University of Minnesota \\ Minneapolis, MN 55455, USA
}

\begin{abstract}
Point vortex and vortex blob computations are used to investigate the evolution of the planar and the axisymmetric vortex sheet which initially induce flow past a cylinder and past a sphere respectively. In both cases the sheet develops a singularity at two points in the symmetry plane at a finite time. It rolls up at these points forming a vortex pair in the planar case and a vortex ring in the axisymmetric case. The computations show differences in the shape of the sheet before singularity formation as well as in the time and location at which the singularity appears. The ensuing roll-up is faster, smaller and less symmetric in the axisymmetric case than in the planar case. Furthermore, unlike the planar pair, the axisymmetric ring sheds approximately $25 \%$ of the total vorticity into its wake which then in turn rolls up into a secondary ring. At large times, irregular particle motion appears. This is believed to result from the onset of chaos in a perturbed dynamical system, and to be caused by oscillations in the downstream motion of the vortices.
\end{abstract}

\section{Introduction}

Consider the potential flow around and inside a circular cylinder which is immersed in uniform parallel flow (Fig. 1a). This flow is induced by a cylindrical vortex sheet bound to the cylinder walls. Equivalently, the potential flow around and inside a sphere immersed in uniform parallel flow is induced by an axisymmetric vortex sheet bound to the sphere (Fig. 1b). This paper presents a numerical study of the vortex sheet evolution if the initially cylindrical and spherical sheets are allowed to evolve freely under their own influence. The goal is to determine differences between the planar and the axisymmetric geometries given comparable initial conditions.

In a reference frame fixed at infinity, one may think of a cylinder and a sphere which have been given an impulse in direction normal to their axes, and are immediately dissolved. The vortex sheet remaining in the fluid moves in direction of the impulse. It evolves and develops a singularity at a finite time at two points in the symmetry plane. After the singularity has formed, the sheet rolls up at these points and forms a counterrotating pair of vortices in the planar case and a vortex ring in the axisymmetric case.

We first investigate the behaviour before singularity formation and the structure of the singularity using a discretization of the exact governing equations. Since the vortex sheet is unstable to Kelvin-Helmholtz short wave growth, a Fourier filter [3] is used to prevent the amplification of high wavenumbers introduced by roundoff error. In the planar case, the singularity formation was studied numerically by Rottman and Stansby [9] and a similar problem was studied by Pugh [7]. Their results show that the order of the planar singularity is the same as in the case of the 
singularity that forms in a periodic vortex sheet (Moore [5], Krasny [3], Shelley [11]). It is not clear a priori that the axisymmetric case behaves similar to the planar case. The results presented here show that the time and position at which the singularity appears are different.

Next, we investigate the long time behaviour of the flow past singularity formation. At these times, a discretization of the exact equations does not converge and the governing equations need to be regularized. We use the vortex blob method in which a smoothing parameter $\delta$ is introduced into the governing equations and obtain information about the sheet in the limit as $\delta \rightarrow 0$. Winckelmans et al. [13] computed the roll-up of the initially spherical sheet as a test problem for a fully $3 \mathrm{D}$ vortex method. Comparison of such fully $3 \mathrm{D}$ solutions with the axisymmetric computations presented here can be used to distinguish axisymmetric from fully $3 \mathrm{D}$ effects and instabilities.

The paper is organized as follows. Section 2 presents the initial conditions and the discretization of the governing equations. Section 3 presents results before and near singularity formation and Section 4 presents aspects of the long time behaviour of the flow. The results are summarized in Section 5 .

\section{Governing equations}

\subsection{Initial conditions}

Let $(x, y, z)$ be a Cartesian coordinate system such that the initial vortex sheets are centered at the origin and the $z$-axis equals the axis of the cylinder. The flow is at rest at infinity and the impulsively given initial velocity is $(U, 0,0)$. By symmetry, both the planar and the axisymmetric sheets are fully described by their crossection with the upper half of the symmetry plane, $z=0, y \geq 0$. This curve is $(x(\alpha, t), y(\alpha, t))$, where the Lagrangian parameter $\alpha$ is chosen to equal arclength at time zero. The initial conditions are

$$
x(\alpha, 0)=\cos \alpha \quad, \quad y(\alpha, 0)=\sin \alpha \quad, \quad \Gamma(\alpha)=-\frac{1}{2}(\cos \alpha+1) \quad, \quad 0 \leq \alpha \leq \pi .
$$

Here, $\Gamma$ is the Lagrangian circulation about the portion of the sheet bounded by the foremost point $(x(0, t), y(0, t))$ and $(x(\alpha, t), y(\alpha, t))$. The vortex sheet strength is $\Gamma^{\prime}(\alpha)=\frac{1}{2} \sin \alpha$. All variables are nondimensionalized with respect to the initial radius and the total circulation in the upper symmetry plane, $\kappa=\Gamma(\pi)$. With this nondimensionalization, the given velocity $U$ is

$$
U_{2 D}=1 / 4 \quad, \quad U_{3 D}=1 / 3
$$

where $2 D$ and $3 D$ refers to the planar and axisymmetric case respectively.

\subsection{Numerical method up to singularity formation}

The vortex sheet motion in the upper symmetry plane is governed by

$$
\mathbf{u}(x, y)=P V \int_{0}^{\pi} \mathbf{u}(x, y ; \widetilde{x}, \widetilde{y}) \Gamma^{\prime}(\widetilde{\alpha}) d \widetilde{\alpha}
$$

Here $x=x(\alpha), y=y(\alpha), \widetilde{x}=x(\widetilde{\alpha}), \widetilde{y}=y(\widetilde{\alpha})$ and $\mathbf{u}(x, y ; \widetilde{x}, \widetilde{y})=(u, v)$ is the velocity at $(x, y)$ induced by a pair of point vortices at $(\widetilde{x}, \pm \widetilde{y})$ and a circular filament at $(\widetilde{x}, \widetilde{y})$ in the planar and axisymmetric case respectively. The velocities are given by derivatives of the respective streamfunctions

$$
\begin{aligned}
& u_{2 D}=\frac{d \psi}{d y}, v_{2 D}=-\frac{d \psi}{d x}, \psi_{2 D}(x, y ; \widetilde{x}, \widetilde{y})=-\frac{1}{4 \pi} \log \frac{(x-\widetilde{x})^{2}+(y-\widetilde{y})^{2}}{(x-\widetilde{x})^{2}+(y+\widetilde{y})^{2}}, \\
& u_{3 D}=\frac{1}{y} \frac{d \psi}{d y}, v_{3 D}=-\frac{1}{y} \frac{d \psi}{d x}, \psi_{3 D}(x, y ; \widetilde{x}, \widetilde{y})=\frac{1}{4 \pi} \int_{0}^{2 \pi} \frac{y \widetilde{y} \cos \theta}{\rho} d \theta
\end{aligned}
$$


where $\rho^{2}=(x-\widetilde{x})^{2}+y^{2}+\widetilde{y}^{2}-2 y \widetilde{y} \cos \theta$. The velocities are discontinuous across the vortex sheet. The integrand in (3) is singular and the principal value effectively assigns to the sheet the average of the velocities on either side of it.

To compute the vortex sheet motion the vortex sheets are discretized by a finite number of (straight or circular) vortex filaments at $\left(x_{j}, y_{j}\right)$ corresponding to $\alpha_{j}=j \pi / N$. The velocity (3) of the filaments is approximated by

$$
\frac{d x_{j}}{d t}=\sum_{\substack{k=0 \\ k \neq j}}^{N} u_{j k} \Gamma^{\prime}\left(\alpha_{k}\right) \Delta \alpha_{k}+u_{j}^{c} \Gamma^{\prime}\left(\alpha_{j}\right) \Delta \alpha_{j}, \frac{d y_{j}}{d t}=\sum_{\substack{k=0 \\ k \neq j}}^{N} v_{j k} \Gamma^{\prime}\left(\alpha_{k}\right) \Delta \alpha_{k}+v_{j}^{c} \Gamma^{\prime}\left(\alpha_{j}\right) \Delta \alpha_{j} .
$$

Here $\Delta \alpha_{k}$ are the trapezoid weights, $u_{j k}=u\left(x_{j}, y_{j} ; x_{k}, y_{k}\right), v_{j k}=v\left(x_{j}, y_{j} ; x_{k}, y_{k}\right)$ and $u_{j}^{c}, v_{j}^{c}$ approximate the self-induced velocity of the vortex sheet segment corresponding to $\alpha_{j-1 / 2} \leq \alpha \leq \alpha_{j+1 / 2}$. The system (5) of ODE's with initial conditions given by (1) is integrated using the 4th order Runge Kutta method. To prevent the growth of small scales introduced by roundoff error, we apply a Fourier filter in which all Fourier coefficients below a specified cutoff level are truncated and set equal to zero at each timestep [3]. Krasny showed numerically that the filter does not alter the spectrum above the cutoff level. Caflisch et al. [2] have recently shown that the filtered computations converge as the filter level is reduced.

The self-induced velocities of a pair of planar vortex sheet segments and of an axisymmetric vortex sheet segment are approximated by

$$
\begin{aligned}
& u_{j, 3 D}^{c}=\frac{1}{2 \pi}\left[\frac{\Gamma^{\prime \prime}}{\Gamma^{\prime}} \frac{b}{c^{2}}+\frac{1}{2 c^{4}}\left(b^{\prime}\left(a^{2}-b^{2}\right)-2 a^{\prime} a b\right)\right]+\frac{1}{2 y_{j}} \\
& v_{j, 2 D}^{c}=-\frac{1}{2 \pi}\left[\frac{\Gamma^{\prime \prime}}{\Gamma^{\prime}} \frac{a}{c^{2}}-\frac{1}{2 c^{4}}\left(a^{\prime}\left(a^{2}-b^{2}\right)+2 b^{\prime} a b\right)\right] \\
& u_{j, 3 D}^{c}=\frac{1}{2 \pi}\left[\frac{\Gamma^{\prime \prime}}{\Gamma^{\prime}} \frac{b}{c^{2}}+\frac{1}{2 c^{4}}\left(b^{\prime}\left(a^{2}-b^{2}\right)-2 a^{\prime} a b\right)\right]+\frac{1}{4 \pi y_{j}}\left[\log \frac{16 \pi y_{j}}{c \Delta \alpha_{k}}-1+b^{2}\right] \\
& v_{j, 3 D}^{c}=-\frac{1}{2 \pi}\left[\frac{\Gamma^{\prime \prime}}{\Gamma^{\prime}} \frac{a}{c^{2}}-\frac{1}{2 c^{4}}\left(a^{\prime}\left(a^{2}-b^{2}\right)+2 b^{\prime} a b\right)\right]-\frac{a b}{4 \pi c^{2} y_{j}}
\end{aligned}
$$

where $a=\partial x / \partial \alpha, b=\partial y / \partial \alpha, c=a^{2}+b^{2}, a^{\prime}=\partial^{2} x / \partial \alpha^{2}, b^{\prime}=\partial^{2} y / \partial \alpha^{2}$, and all derivatives are evaluated using a spectral method.

The discretization (5), (6) equals a discretization of the sheet by point vortex pairs (planar case) and circular vortex filaments (axisymmetric case) in addition to a correction to better approximate the velocity a vortex sheet segment induces on itself. In the planar case, this corrective term was computed by van de Vooren [12], in the axisymmetric case, by de Bernadinis and Moore [1]. The inclusion of these terms improves the rate of convergence before the singularity formation.

\subsection{Numerical method past singularity formation}

Past the time of singularity formation, a numerical solution to the exact equations does not converge as the computational mesh is refined. To compute the vortex sheet motion at these times it is necessary to regularize the vortex sheet. We use the vortex blob method in which the governing equations are regularized by convolution with a smoothing function. The regularization depends on a parameter $\delta$ and information about the vortex sheet is recovered in the limit $\delta \rightarrow 0$ [4]. The 
regularized equations are

$$
\mathbf{u}^{\delta}(x, y)=\int_{0}^{\pi} \mathbf{u}^{\delta}(x, y ; \widetilde{x}, \widetilde{y}) \Gamma^{\prime}(\alpha) d \alpha
$$

where $\mathbf{u}^{\delta}(x, y ; \widetilde{x}, \widetilde{y})$ is given by derivatives of the regularized streamfunction

$$
\begin{aligned}
& \psi_{2 D}(x, y ; \widetilde{x}, \widetilde{y})=-\frac{1}{4 \pi} \log \frac{(x-\widetilde{x})^{2}+(y-\widetilde{y})^{2}+\delta^{2}}{(x-\widetilde{x})^{2}+(y+\widetilde{y})^{2}+\delta^{2}} \\
& \psi_{3 D}(x, y ; \widetilde{x}, \widetilde{y})=\frac{1}{4 \pi} \int_{0}^{2 \pi} \frac{y \widetilde{y} \cos \theta}{\left(\rho^{2}+\delta^{2}\right)^{1 / 2}} d \theta .
\end{aligned}
$$

The integrand in (7) is nonsingular and is evaluated using the trapezoid rule:

$$
\frac{d x_{j}}{d t}=\sum_{k=0}^{N} u_{j k}^{\delta} \Gamma^{\prime}\left(\alpha_{k}\right) \Delta \alpha_{k} \quad, \quad \frac{d y_{j}}{d t}=\sum_{k=0}^{N} v_{j k}^{\delta} \Gamma^{\prime}\left(\alpha_{k}\right) \Delta \alpha_{k}
$$

As in 2.2, the system of ODE's (9) with initial conditions (1) is integrated using the 4th order Runge Kutta method. For large time computations, the computed vortex sheet roll-up is resolved by inserting points such that the maximum distance between points and the angle consecutive points make with the spiral center remains small.

\section{Behaviour near singularity formation}

Figure 2 shows the computed planar and axisymmetric solution at a sequence of times, using $N=200$. In both cases, the sheet moves to the right in direction of the impulse. The particles near the front of the sheet cause the particles near the rear to reduce in radius, which in turn causes these ones to move faster and the sheet deforms. In the axisymmetric case, the rear moves faster relative to the front than in the planar case and the sheet becomes slightly more narrow. At $\mathrm{t}=2$ the rear of the sheet is almost flat. The curvature in the rear then changes sign and shortly afterwards a cusp develops in the sheet at $t=t_{c}^{N}$. This is the last time shown in Fig. 2.

The time $t_{c}^{N}$ is determined to be the time at which one meshpoint first crosses a neighbouring point from below to above. Figure 3 shows a closeup of the axisymmetric vortex sheet at $t=t_{c}^{N}$, for various values of $N$. It can be seen that the sheet has lost regularity. To investigate the time and position at which the singularity occurs we investigate the time and position of this cusp in the $\operatorname{limit} N \rightarrow \infty$. This limit $t_{c}=\lim _{N \rightarrow \infty} t_{c}^{N}$ defines the time before which the sheet is smooth to the eye and the numerical solution converges. Alternatively, Krasny suggests that the time of singularity formation can be given by the limit $t_{s}=\lim _{N \rightarrow \infty} t_{s}^{N}$. Here, $t_{s}^{N}$ is defined as the time at which the minimum distance between any two points has reached a minimum, $s_{m i n}^{N}$.

Figures 4(a-c) show the behaviour of $t_{c}^{N}, t_{s}^{N}$ and $s_{\text {min }}^{N}$ as $N \rightarrow \infty$. Figures 4(d-f) show the position $\left(x_{c}^{N}, y_{c}^{N}\right)$ and the Lagrangian coordinate $\alpha_{c}^{N}$ at the cusp that has formed at $t=t_{c}^{N}$, for various $N$. Each subplot also shows a least squares quadratic curve approximating the points. The curve indicates that the values are well approximated by an expansion

$$
q^{N} \sim q_{o}+q_{1} / N+q_{2} / N^{2}+\cdots
$$

We extrapolate the quadratic curve to obtain the limit $q_{o}=\lim _{N \rightarrow \infty} q^{N}$. The limiting values are recorded in Table I. Notice that the limiting times $t_{c}$ and $t_{s}$ agree to within $1 \%$. 
Table I: Values of the indicated quantities in the limit $N \rightarrow \infty$. The values are obtained by quadratic extrapolation of the data in Fig. 4 to $1 / N=0$.

\begin{tabular}{|l|c|c|c|c|c|c|}
\hline & $t_{c}$ & $t_{s}$ & $s_{\min }$ & $x_{c}$ & $y_{c}$ & $\alpha_{c}$ \\
\hline Planar & 2.339 & 2.340 & 0.000016 & -0.245 & 0.453 & $0.750 \pi$ \\
\hline Axisymmetric & 2.222 & 2.222 & 00.000427 & 0.046 & 0.512 & $0.720 \pi$ \\
\hline
\end{tabular}

We observe some differences between the axisymmetric and the planar case. The time $t_{c}$ is slightly smaller in the axisymmetric case. The coordinates $x_{c}, y_{c}, \alpha_{c}$ of the point at which the singular behaviour appears are different. The value of $x_{c}$ is larger in the axisymmetric case, since the axisymmetric sheet travels faster. The singularity appears at a larger y-coordinate and at a correspondingly lower value of the parameter $\alpha_{c}$ in the axisymmetric case. We remark that the values for $N$ were chosen such that a meshpoint fell close to $\alpha_{c}=0.75 \pi, 0.72 \pi$ respectively. If $\alpha_{c}$ fell at equal distances in between 2 meshpoints, this was reflected in large deviations in the measured quantities a-e, which unnecesarily clouded the picture. The values of $N$ used were $N=24,32,48,56,68,84,100,148,200,248,300,400$ in the planar case, and $N=25,32,43,50,57,68,82,100,150,200,250,300,400$ in the axisymmetric case. While there are clear differences in the time and position at which the planar and axisymmetric singularities occurs, preliminary analysis of the Fourier coefficients indicates that the order of the singularity is the same in both cases. The resolution of this issue is in preparation and will be presented elsewhere.

A view of what happens briefly after singularity formation is given in Fig. 5. The solution is shown at $t-t_{c} \approx 0.634$, computed with $N=1000$ and $\delta=0.1$. The sheet has rolled up near the point of singularity formation.

\section{Behaviour at large times}

Figures $6-8$ show the computed solution at large times past singularity formation. The computations are performed with $\delta=0.2$. The planar case is shown in Fig. 6. The sheet rolls up near $\alpha=\alpha_{c}$ into a double spiral and forms a vortex pair. At $t=20$, the outer turn of the sheet lags behind the vortex core and forms a 'tail' at $t=30,40$. However, most of the particles in this tail are entrained back into the fluid moving with the ring. At $t=120$, the parts of the sheet near the axis that form a 'tail' carry almost no vorticity. This is shown in Fig. 7a. The figure shows vorticity contours and streamlines of the planar sheet at $t=120$. A bubble of fluid moves with the vortex pair, and there is essentially zero vorticity outside this bubble.

The axisymmetric case is shown in Fig. 8. The sheet rolls up and forms a vortex ring. The ring travels faster and is noticeably smaller than the corresponding planar vortex pair. As in the planar case, an outer turn is seen to lag behind the spiral rollup at $t=10$. At time $t=20$, the tail that has formed is much larger than in the planar case. At later times, it is not entrained back into the ring, but is left behind the primary ring. After approximately $t=30$ there is about $25 \%$ of vorticity in this tail. This portion of the sheet rolls up and forms a secondary ring, which travels slower than the first. In turn, this secondary ring has its own tail, which contains about $7 \%$ of the total vorticity (or approximately $25 \%$ of the vorticity in the primary tail). Figure $7 \mathrm{~b}$ shows vorticity contours of the axisymmetric sheet at $t=80$, clearly showing two distinct regions of vorticity. Figure $7 \mathrm{~b}$ also shows the streamlines in a reference frame moving with an average velocity. The primary ring velocity is close to the average velocity whereas the secondary ring moves slower and therefore this reference frame does not show the bubble of fluid moving with the smaller ring.

Figure 9 shows the computed solution with decreasing values of the smoothing parameter $\delta=$ 
$0.2,0.1,0.05$. The amount of vorticity shed in the axisymmetric case remains close to $25 \%$. The figure shows that the essential features observed in Figs. 6 and 7 remain basically unchanged and are not an artifact of the smoothing parameter.

Figure 9f shows small waves appearing on the inner portion of the tail of the axisymmetric sheet. Here, the sheet does not stretch enough to counteract the growth of roundoff error and the Kelvin-Helmholtz instability can be observed. This irregular particle behaviour is an effect of finite machine precision and would decrease with higher precision. In Winckelmans et al.'s [13] computations this axisymmetric instability is observed before the growth of unstable azimuthal waves.

There is another kind of irregular particle motion which is not a numerical artifact and is shown in Fig. 10. Figures 10(a-c) show the development of the irregular motion near the rear of the planar sheet, at times $t=90,100,110$. This irregular motion is also observed in the axisymmetric sheet in Fig. 7, at $t=80$. Figures $10(\mathrm{~d}-\mathrm{e})$ show a closeup of the core of the planar sheet, at times $t=120,130,140$. Gaps are seen that develop between the spiral turns. These gaps grow in time as they rotate with the flow around the core. This behaviour is documented in more detail in [6], for a related case of vortex sheet roll-up from initially flat vortex sheets.

To explain the occurrence of the behaviour observed in Fig. 10, let us return to the streamlines shown in Fig. 8. The streamlines show two hyperbolic stagnation points in the front and in the rear of the bubble of fluid moving with the vortex. Rom-Kedar et al. [8] study the dynamics of a pair of point vortices whose motion is perturbed by superposing a periodic strain-field. The streamlines in that case look similar to the ones in Fig. 8. Rom-Kedar et al. show that the perturbation causes the stable and unstable manifolds associated with the front and rear stagnation point to intersect transversely. This in turn causes irregular entrainment and detrainment of fluid particles into and out of the bubble of fluid. The perturbation also affects the region near the core. Here, invariant circles in the Poincaré map of the original unperturbed flow break up into a finite number of periodic orbits. Within and nearby these orbits the motion becomes chaotic and resonance bands form. Similar observations were made by Shariff et al. [10], who studied the dynamics of elliptic vortex rings which oscillate periodically in time. The irregular motion of particles near the rear and near the core that we see in Fig. 10 thus reflects the dynamics of such a perturbed system.

To give some evidence for a quasi-periodic perturbation in our computed flow, Fig. 11 shows the y-coordinate $Y_{m}$ and velocity $U_{m}$ of the vorticity maximum of the vortex pair and the vortex ring vs. time. Both these quantities oscillate in time. These oscillations may be the origin for the irregular particle motion in Fig. 10.

\section{Summary of results}

We presented a numerical study of the evolution of a cylindrical and a spherical vortex sheet. The vortex sheets evolve and develop a singularity which was shown to occur earlier and with larger $\mathrm{y}$-coordinate in the axisymmetric case. Past the time of singularity formation the vortex sheets roll up and form a vortex pair (planar case) and a vortex ring (axisymmetric case). The axisymmetric roll-up is smaller and faster than the planar one. Furthermore, unlike the planar vortex pair, the axisymmetric ring sheds a significant amount of vorticity into the rear, where it rolls up to form a secondary ring. Irregular particle motion is obseved near the rear and near the core of the vortices and is conjectured to represent the chaotic motion previously observed in slightly perturbed dynamical systems. 


\section{References}

[1] Bernadinis, B. de and Moore, D.W., "A Ring-Vortex Representation of an Axi-Symmetric Vortex Sheet," In Studies of Vortex Dominated Flows, Proc. Symp. July 9-11, 1985, NASA Langley Research Center, edited by M.Y. Hussaini and M.D. Salas, Springer, 1986.

[2] Caflisch, R.E., Hou, T.Y. and Lowengrub, J., "Almost Optimal Convergence of the Point Vortex Method for Vortex Sheets using Numerical Filtering," to be published in Math. Comp., 1995.

[3] Krasny, R., "A Study of Singularity Formation in a Vortex Sheet by the Point-Vortex Approximation," J. Fluid Mech., 167, pp. 65, 1986.

[4] Krasny, R., "Desingularization of Periodic Vortex Sheet Roll-Up," J. Comp. Phys., 65, pp. 292, 1986.

[5] Moore, D.W., "The Spontaneous Appearance of a Singularity in the Shape of an Evolving Vortex Sheet," Proc. R. Soc. Lond. A, 365, pp. 105, 1979.

[6] Nitsche, M. and Krasny, R., "Comparison of Planar and Axisymmetric Vortex Sheet Roll-Up," in preparation, 1996.

[7] Pugh, D.A., "Development of Vortex Sheets in Boussinesq Flows-Formation of Singularities," Ph.D. Thesis, Imperial College of Science and Technology, London, 1989.

[8] Rom-Kedar, V., Leonard, A. and Wiggins, S., "An Analytical Study of Transport, Mixing and Chaos in an Unsteady Vortical Flow," J. Fluid Mech., 214, pp. 347, 1990.

[9] Rottman, J.W. and Stansby, P.K., "On the $\delta$-Equation for Vortex Sheet Evolution," J. Fluid Mech., 247, pp. 527, 1993.

[10] Shariff, K., Leonard, A. and Ferziger, J.H., "Dynamics of a Class of Vortex Rings," NASA TM-10225\%, 184, pp. 123, 1989.

[11] Shelley, M., "A Study of Singularity Formation in Vortex Sheet Motion by a Spectrally Accurate Vortex Method," J. Fluid Mech., 244, pp. 493, 1992.

[12] van de Vooren, A.I., "A Numerical Investigation of the Rolling Up of Vortex Sheets," Proc. $R$. Soc. Lond. A, 373, pp. 67-80, 1980.

[13] Winckelmans, G.S., Salmon, J.K., Leonard, A. and Warren, M.S., "Three-Dimensional Vortex Particle and Panel Methods: Fast Tree-Code Solvers with Active Error Control for Arbitrary Distributions/Geometries," Proc. Forum on Vortex Methods for Eng. Appl., Sandia National Labs., Albuquerque, NM, Feb. 22-24, 1995. 
a)

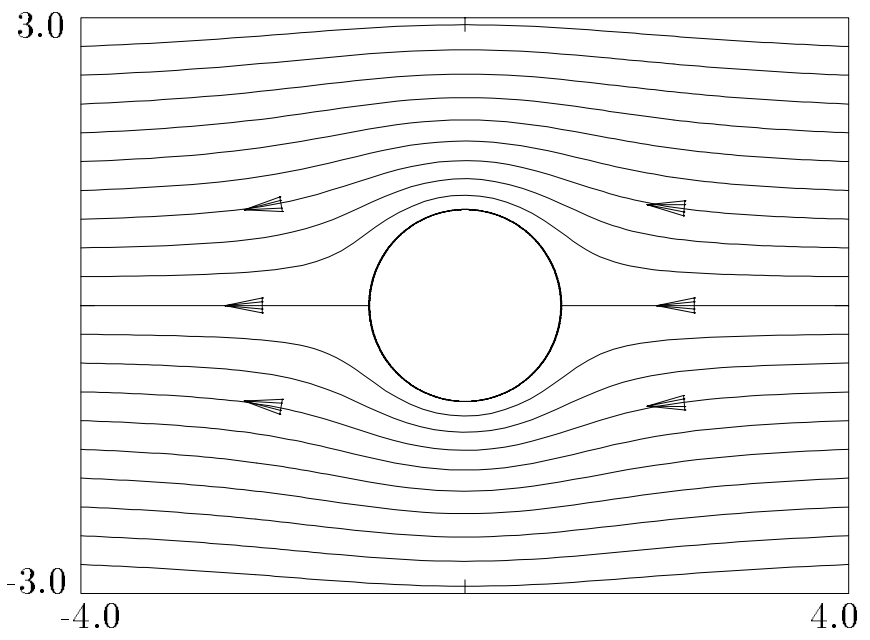

b)

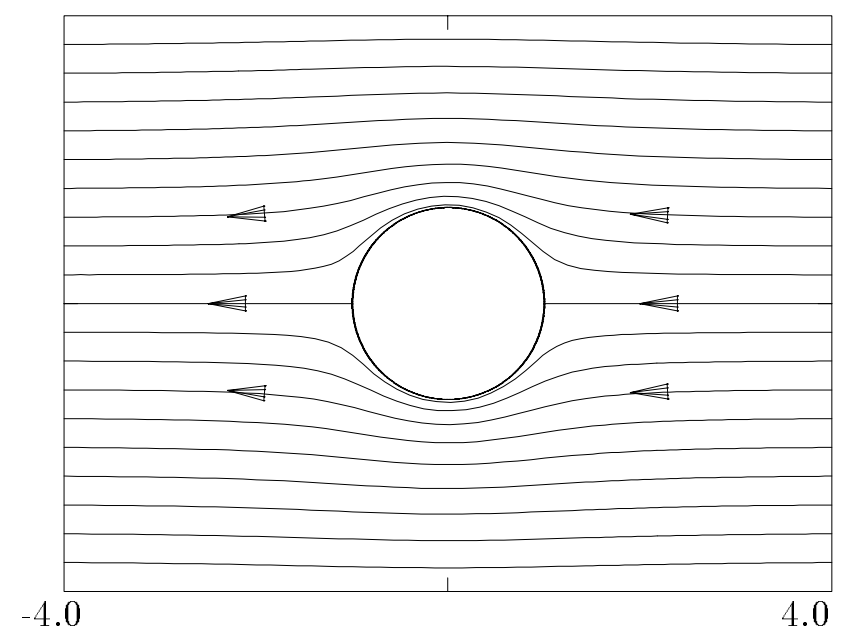

Figure 1: a) Potential flow past a circular cylinder. b) Potential flow past a sphere. The flow is uniform and parallel at infinity and zero inside the closed streamlines.
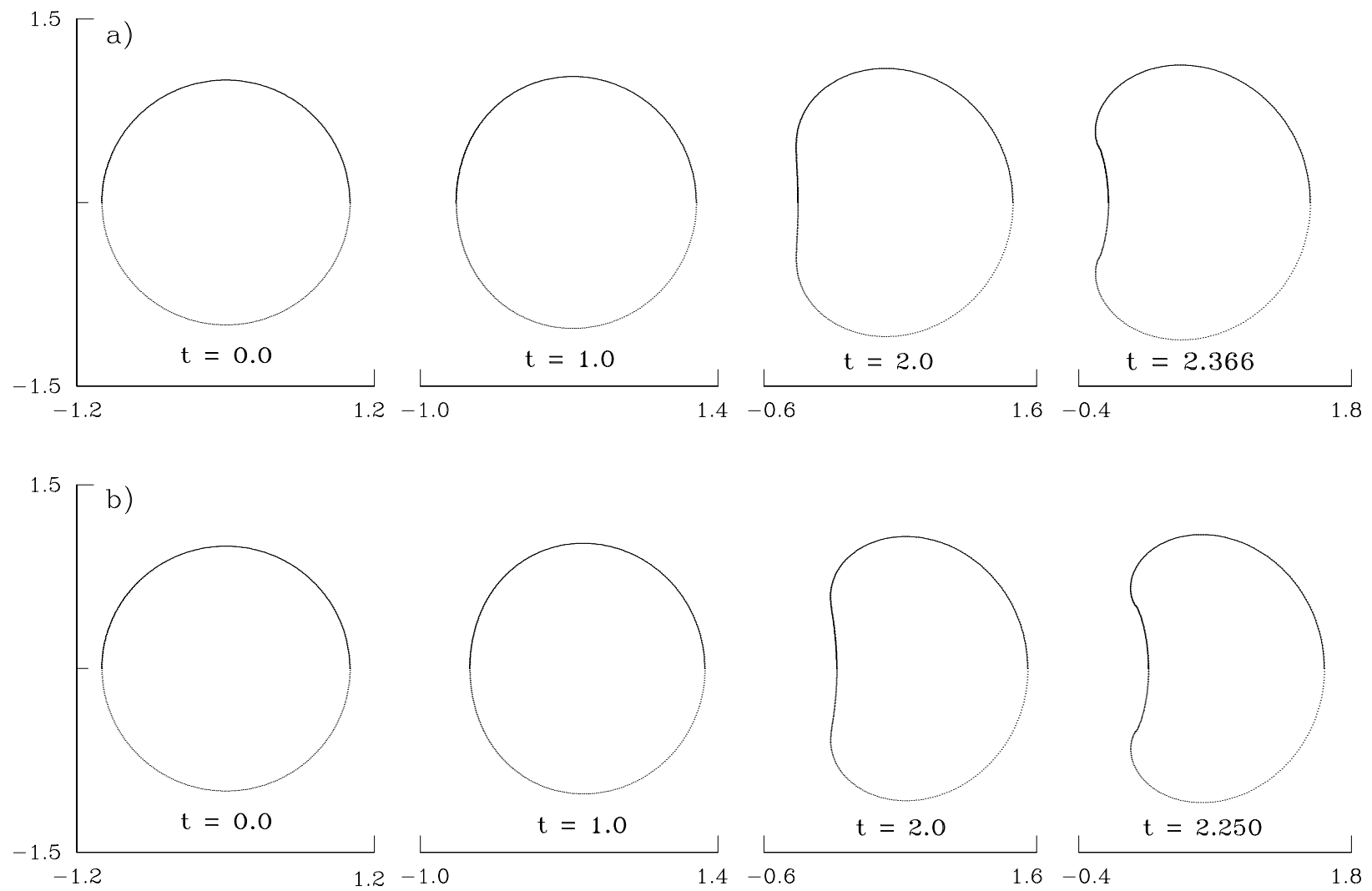

Figure 2: Computed vortex sheet at the indicated times, with $N=200$ and $\delta=0.0$. The last time shown is $t=t_{c}^{N}$. a) Planar sheet. b) Axisymmetric sheet. 
a)

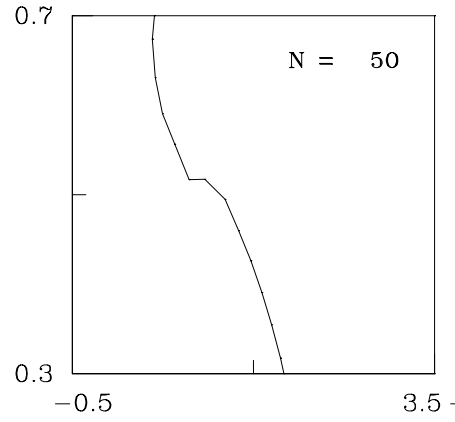

b)

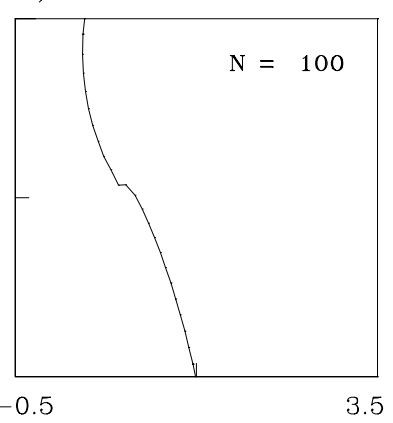

c)

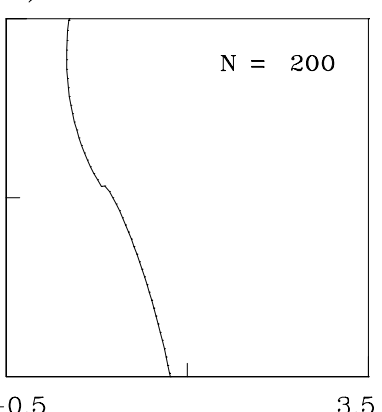

d)

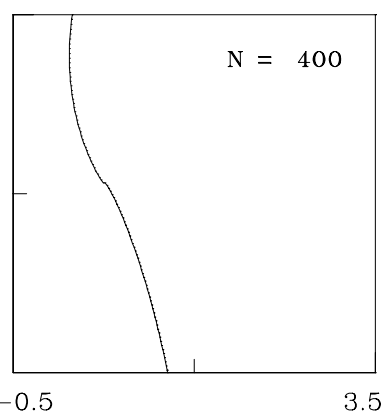

Figure 3: Closeup. Axisymmetric vortex sheet at the time $t=t_{c}^{N}$ at which one point first crosses from below to above another point. a) $N=50, t_{c}^{50}=2.329$, b) $N=100, t_{c}^{100}=2.277$, c) $N=200$, $t_{c}^{200}=2.250$, d) $N=400, t_{c}^{400}=2.234$.
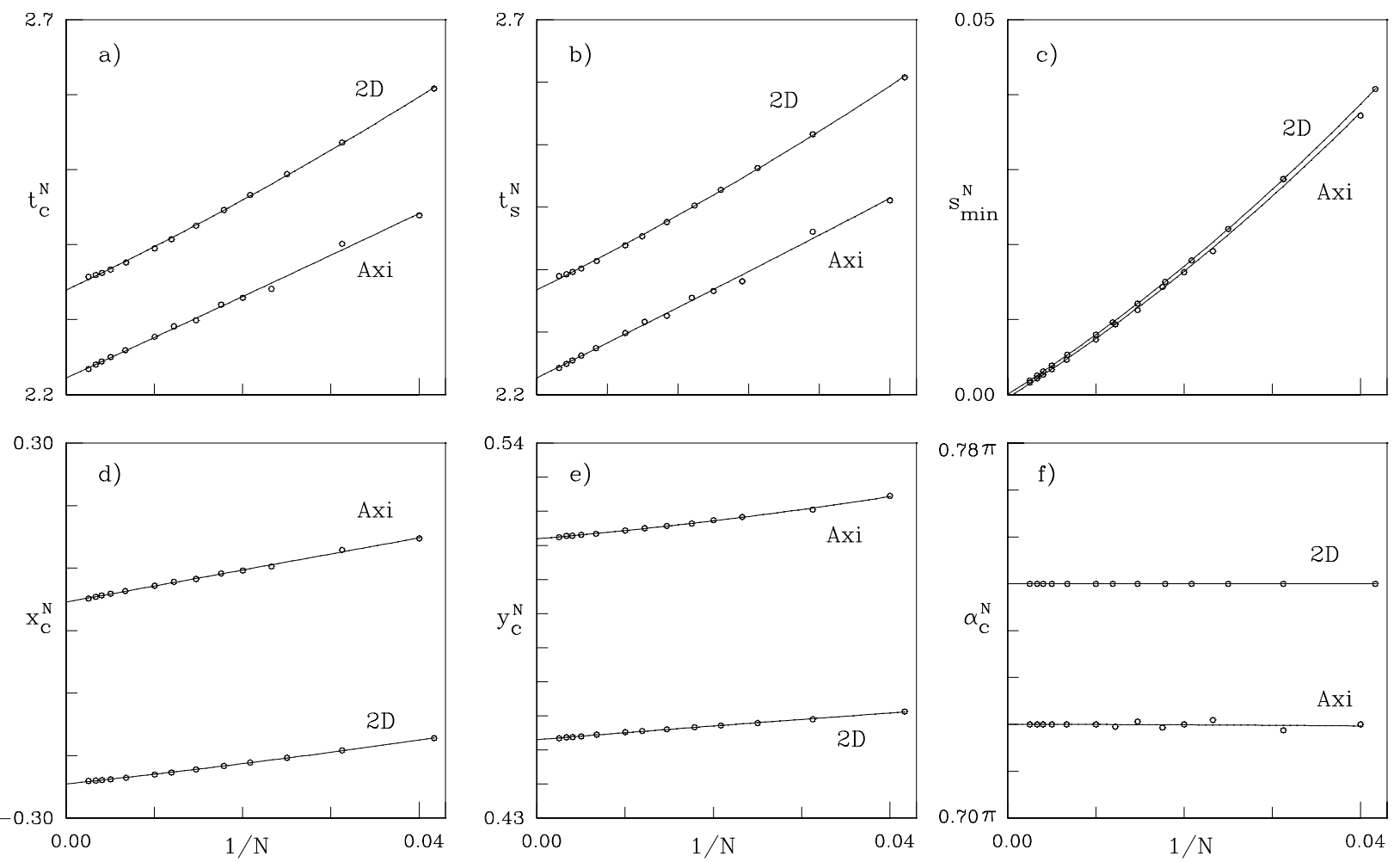

Figure 4: Quantities describing singularity formation, vs. 1/N. A quadratic least squares curve is fit to the data. a) $t_{c}^{N}$, b) $t_{s}^{N}$, c) $s_{m i n}^{N}$, d) $x_{c}^{N}$ e) $y_{c}^{N}$ f) $\alpha_{c}^{N}$ 

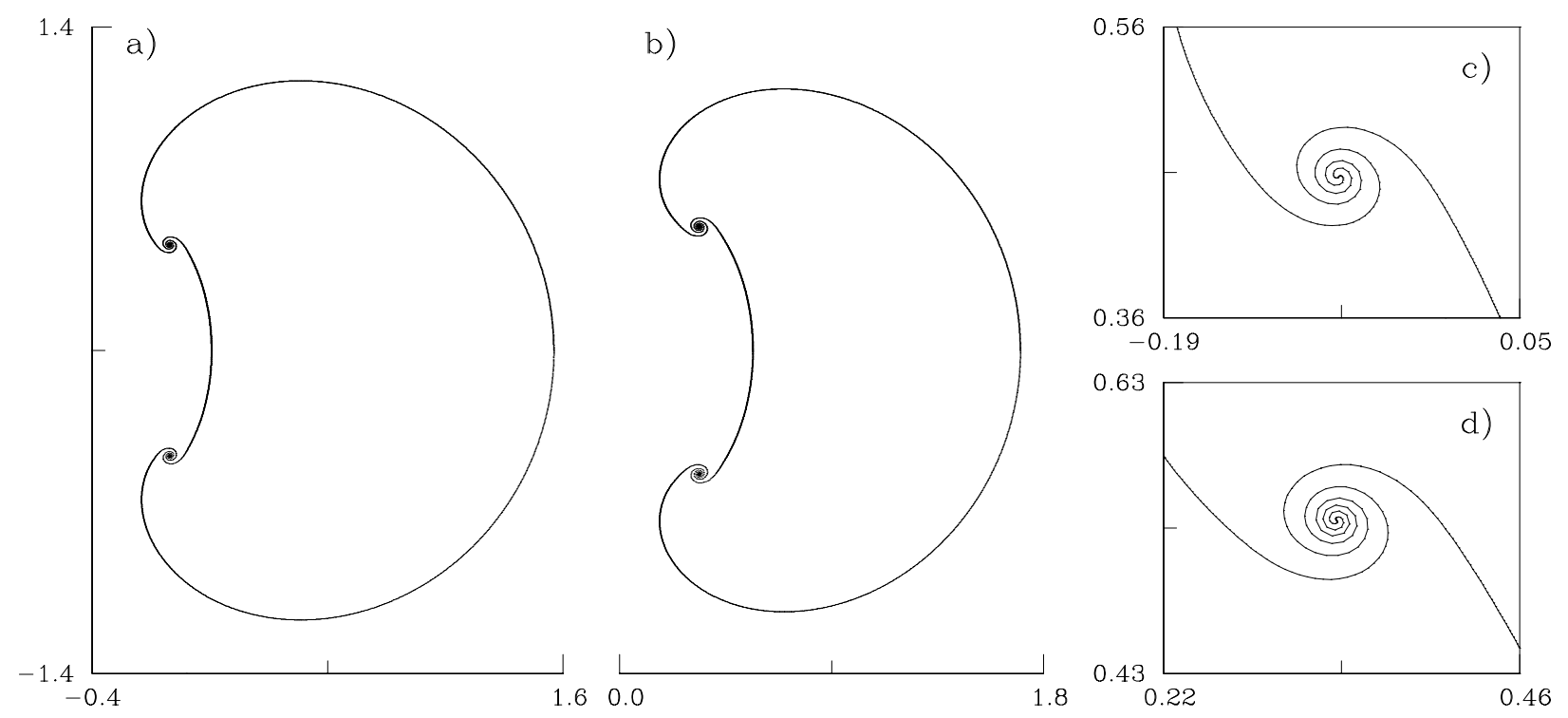

Figure 5: Computed vortex sheet after singularity formation, with $\delta=0.1, N=1000$. a,c) Planar sheet at $t=3.0 . \mathrm{b}, \mathrm{d}$ ) Axisymmetric sheet at $t=2.9$. The times were chosen so that $t-t_{c} \approx 0.7$ is approximately the same time in both cases.

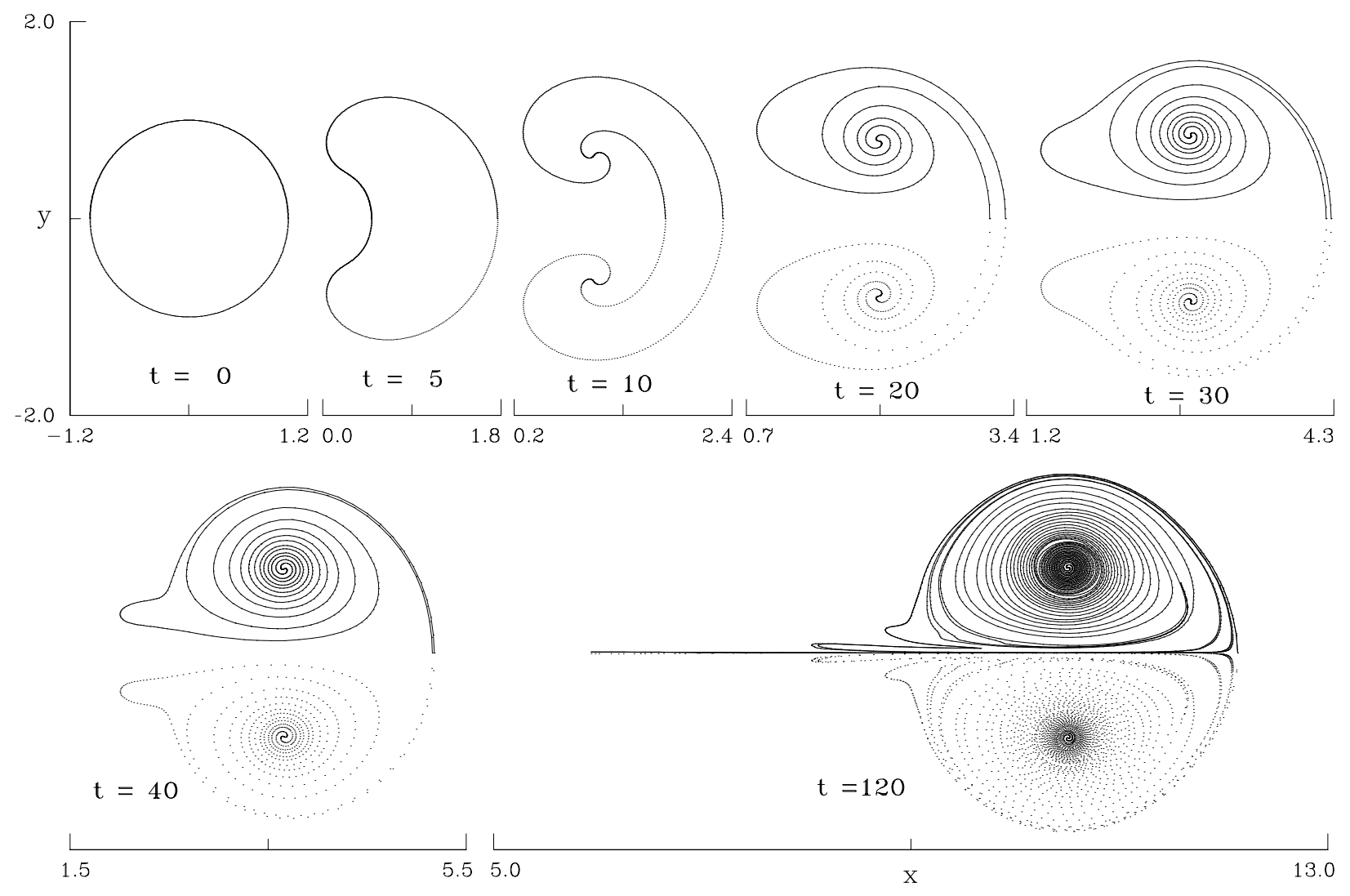

Figure 6: Computed planar vortex sheet at the indicated times, with $\delta=0.2$. 
a)
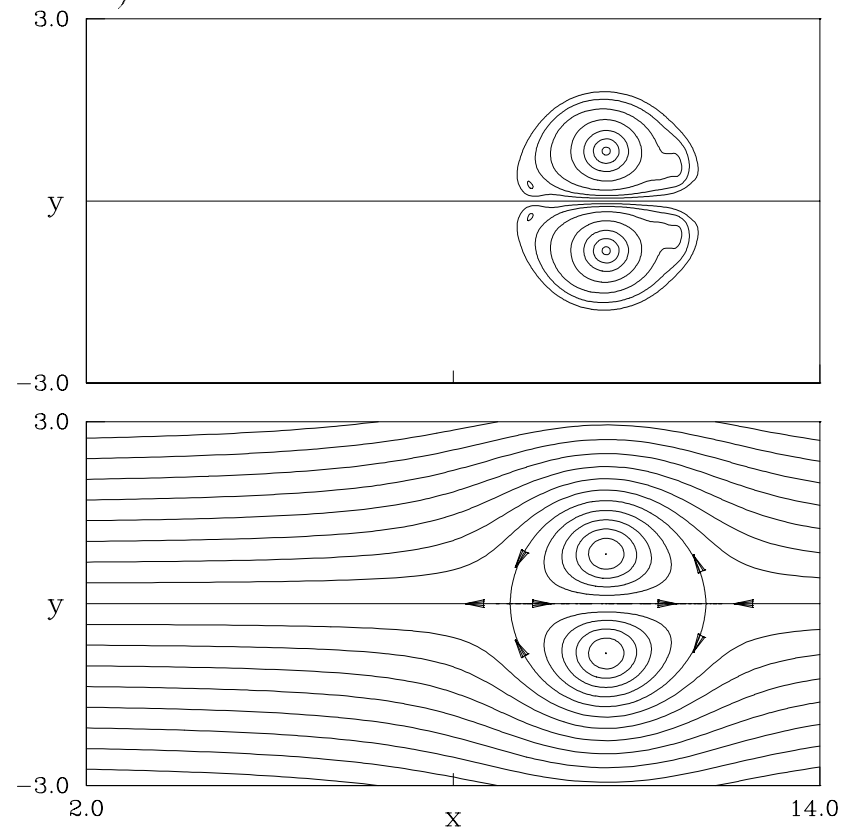

b)
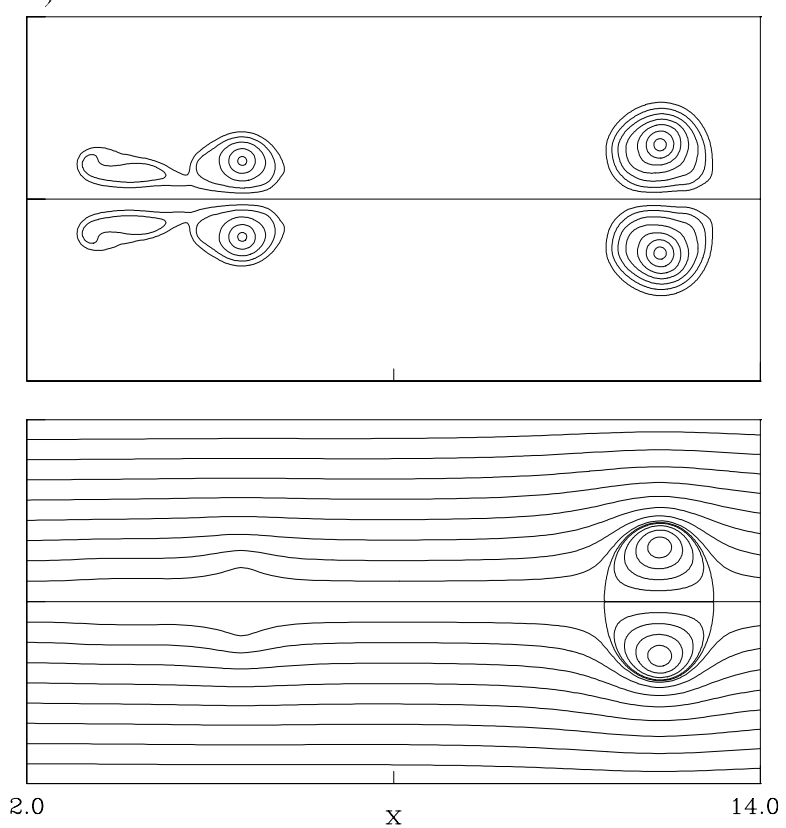

Figure 7: Vorticity contours and streamlines for the computed solution with $\delta=0.2$. a) Planar sheet at $t=120$. Axisymmetric sheet at $t=80$. The vorticity levels shown in both a) and b) are $\omega=2,1,1 / 2,1 / 4,1 / 8,1 / 16,1 / 32$. 

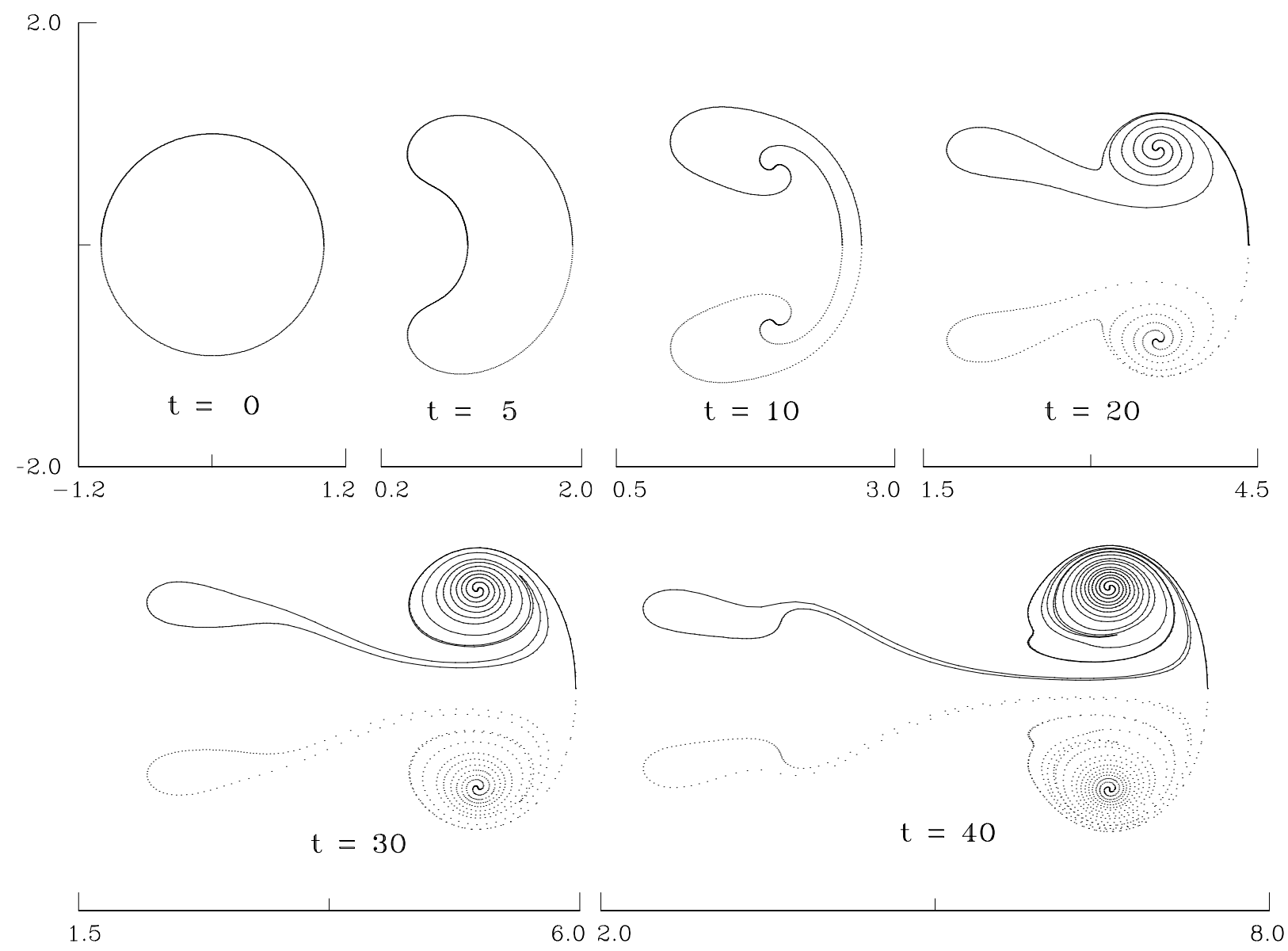

1.5

6.02 .0

8.0

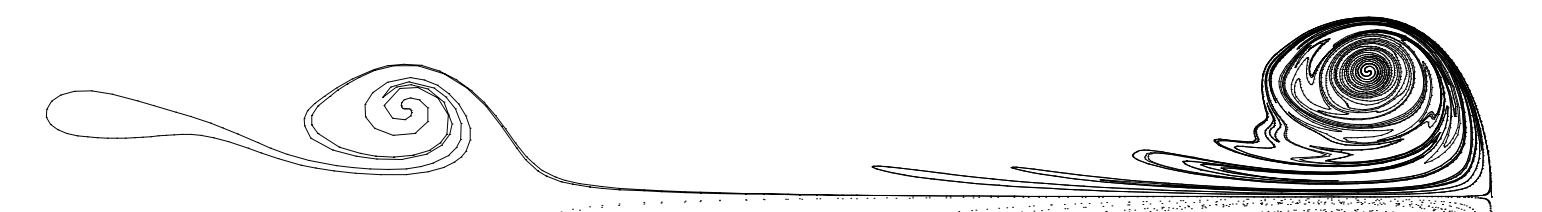

$t=80$

2.7

Figure 8: Computed axisymmetric vortex sheet at the indicated times, with $\delta=0.2$. 

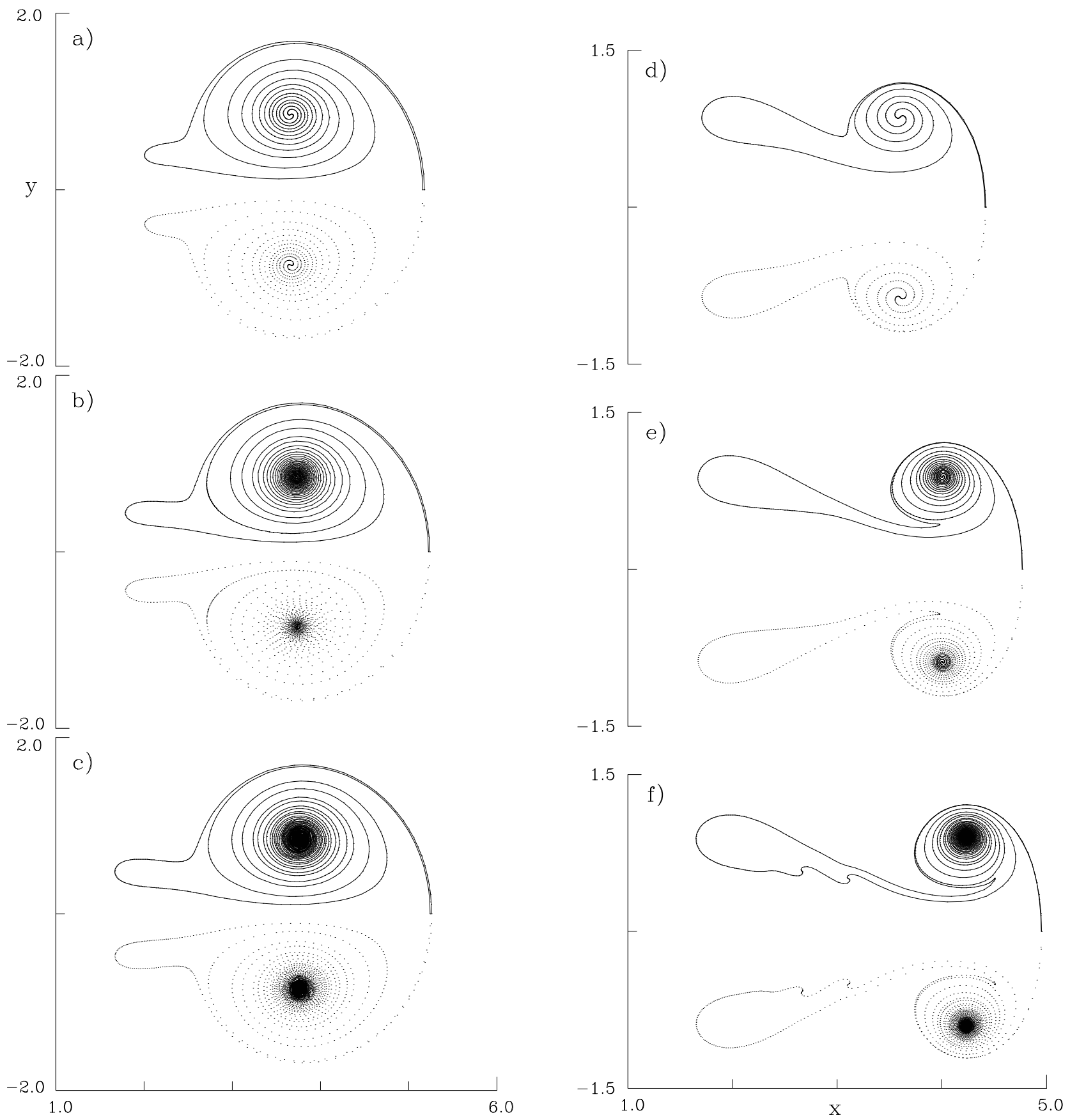

Figure 9: Planar sheet at $t=40$, with a) $\delta=0.2$, b) $\delta=0.1$, c) $\delta=0.05$. Axisymmetric sheet at $t=20$, with d) $\delta=0.2$, e) $\delta=0.1$, f) $\delta=0.05$. 

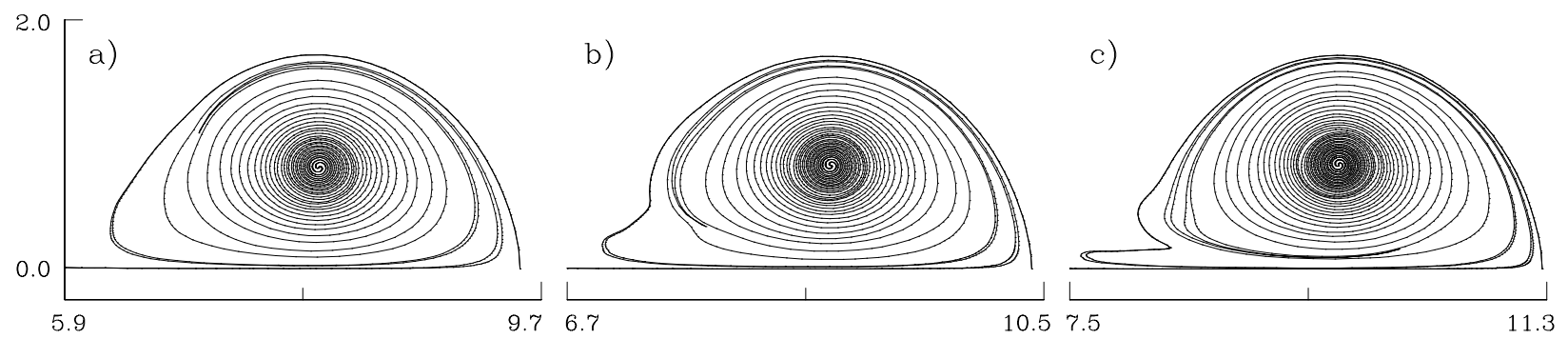

d)

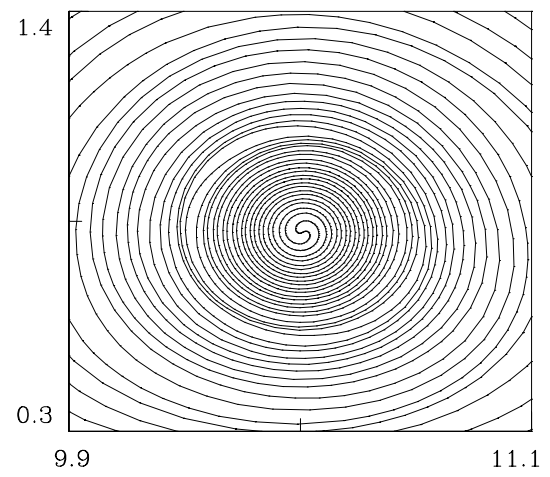

e)

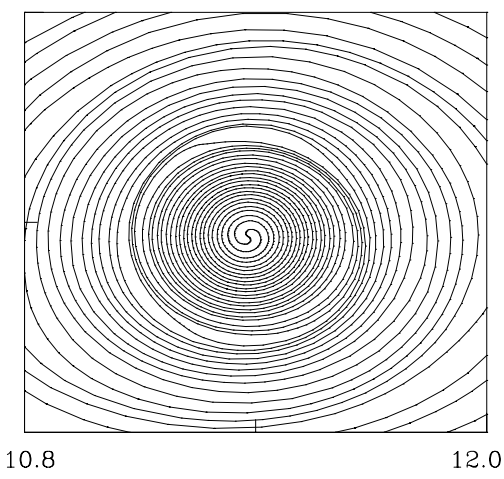

f)

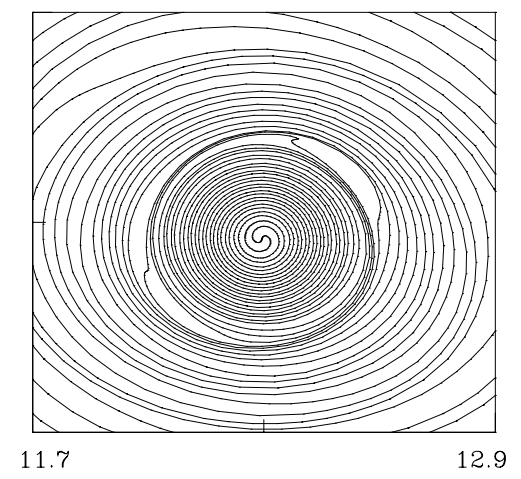

Figure 10: Planar vortex sheet computed with $\delta=0.2$, showing the onset of irregular behaviour near the rear stagnation point and near the core. a) $t=90$, b) $t=100$ c) $t=110 \mathrm{~d}$ ) $t=120$, e) $t=130, \mathrm{f}) t=140$.

a)
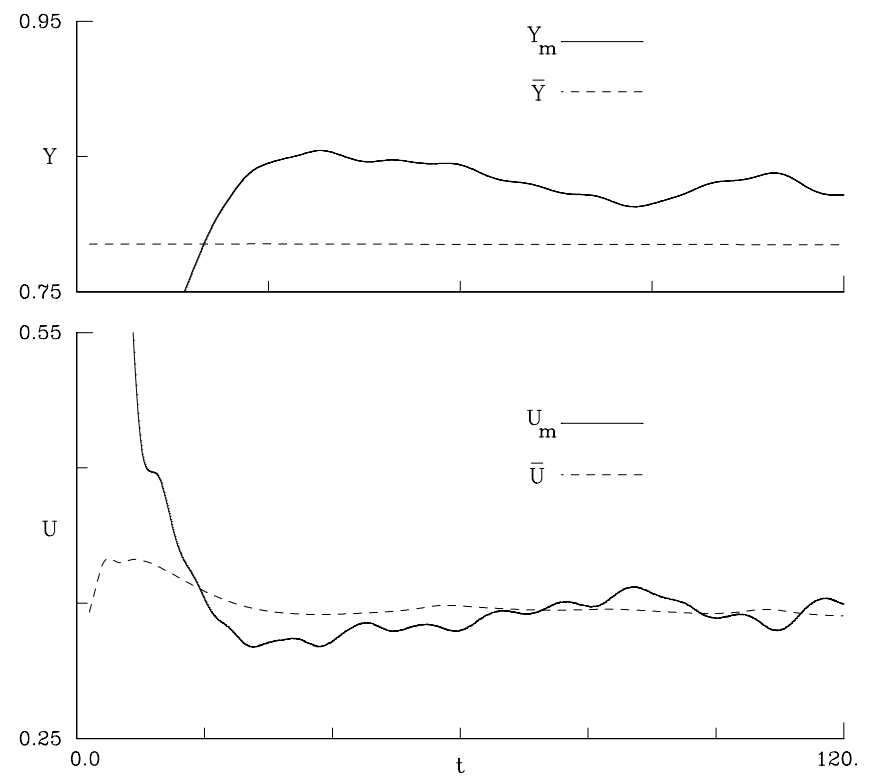

b)
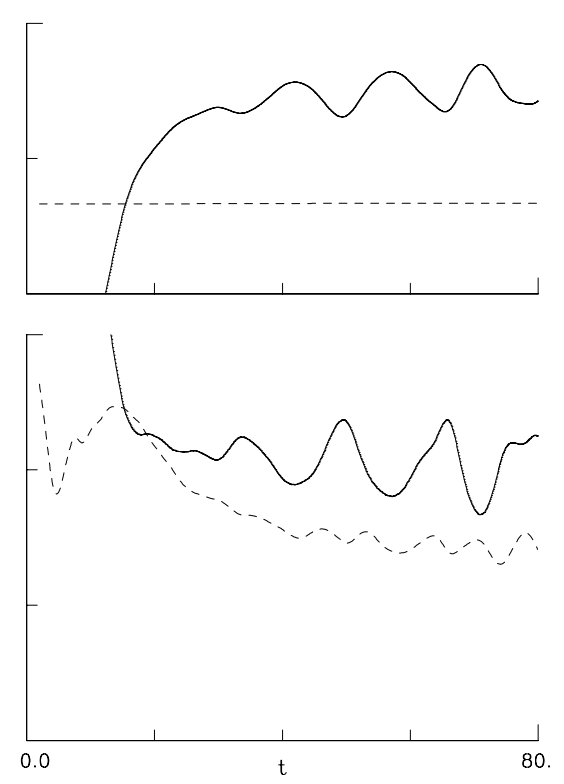

Figure 11: Characteristic distance $Y$ and translation velocity $U$ of the rolled-up vortex sheets. a) Planar vortex pair. b) Axisymmetric vortex ring. Both the average values $\bar{Y}, \bar{U}$ and the values at the vorticity maximum $Y_{m}$ and $U_{m}$ are shown. 